\title{
Nano-mechanical Phenotype as a Promising Biomarker to Evaluate Cancer Development, Progression, and Anti-cancer Drug Efficacy
}

\author{
Soyeun Park \\ College of Pharmacy, Keimyung University, Daegu, Korea
}

\begin{abstract}
Since various bio-mechanical assays have been introduced for studying mechanical properties of biological samples, much progress has been made in cancer biology. It has been noted that enhanced mechanical deformability can be used as a marker for cancer diagnosis. The relation between mechanical compliances and the metastatic potential of cancer cells has been suggested to be a promising prognostic marker. Although it is yet to be conclusive about its clinical application due to the complexity in the tissue integrity, the nano-mechanical compliance of human cell samples has been evaluated by several groups as a promising marker in diagnosing cancer development and anticipating its progression. In this review, we address the mechanical properties of diverse cancer cells obtained by atomic force microscopy-based indentation experiments and reiterate prognostic relations between the nano-mechanical compliance and cancer progression. We also review the nano-mechanical responses of cancer cells to the anti-cancer drug treatment in order to interrogate a possible use of nano-mechanical compliance as a means to evaluate the effectiveness of anti-cancer drugs.

(J Cancer Prev 2016;21:73-80)
\end{abstract}

Key Words: Elastic modulus, Atomic force microscopy, Neoplasm metastasis, Chemotherapeutic anticancer agents

\section{INTRODUCTION}

The physical interaction with surrounding microenvironments and the negotiation of biochemical signaling cascade play a key role in cancer formation and metastasis. ${ }^{1}$ The interaction with the extracellular matrix and intra/extra-vasation requires that cancer cells undergo mechanical deformations in response to the external stress by remodeling their actin cytoskeletons. Over the past decade, mechanical properties of biological cells have been investigated by using several tools such as micropipette aspiration, optical stretchers, magnetic twisting cytometry, and microplate stretcher. ${ }^{2}$ Advantages, such as the adaptability to physiological environments and the ability to measure forces with a pico-newton sensitivity from precisely controlled local domains make atomic force microscopy (AFM) the most widely used tool for characterizing mechanical properties of cells (Fig. 1).

Many studies have found that cancer cells are mechanically more compliant ('softer') than normal cells, and thus the enhanced mechanical compliance can serve as a promising marker for cancer diagnosis and metastasis. ${ }^{3-5}$ However, several studies have also shown reverse or non-significant correlations between the mechanical compliance and cancer metastasis. ${ }^{6-8}$ During the metastatic progression, cancer cells encounter various microenvironments where they doom to adjust mechanical properties such as motility, adhesion, and mechanical compliance. Cancer cells' ability to adjust mechanical properties at each step of metastasis plays a major role in cancer metastasis. Recent studies have suggested that the combined mechanical signatures including motility and adhesion can resolve the unequivocal issue raised by the sole use of mechanical compliance in cancer prognosis. ${ }^{7.9}$ In regard to this multipronged approach, AFM is a superior tool because it can be used to investigate changes in morphology and the mechanical compliance of cancer cells caused by the drug treatment (Fig. 1A), ${ }^{10,11}$ single-molecule

Received May 17, 2016, Revised June 4, 2016, Accepted June 6, 2016

Correspondence to: Soyeun Park

College of Pharmacy, Keimyung University, 1095 Dalgubeol-daero, Dalseo-gu, Daegu 42601, Korea

Tel: +82-53-580-5164, Fax: +82-53-580-6654, E-mail: sypark20@kmu.ac.kr, ORCID: Soyeun Park, http://orcid.org/0000-0003-3989-9079

Copyright (c) 2016 Korean Society of Cancer Prevention

(c) This is an Open Access article distributed under the terms of the Creative Commons Attribution Non-Commercial License (http://creativecommons.org/licenses/by-nc/4.0) which permits unrestricted non-commercial use, distribution, and reproduction in any medium, provided the original work is properly cited. 
A

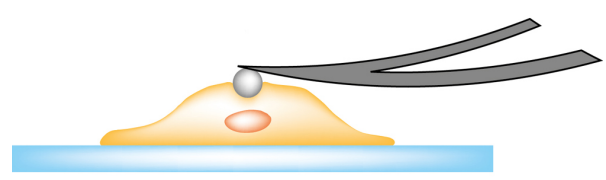

C

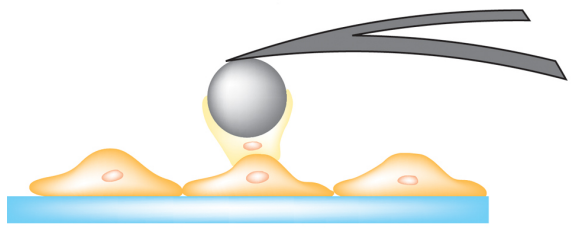

B

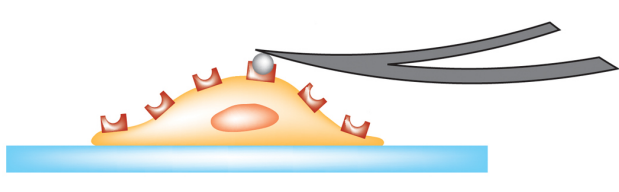

D

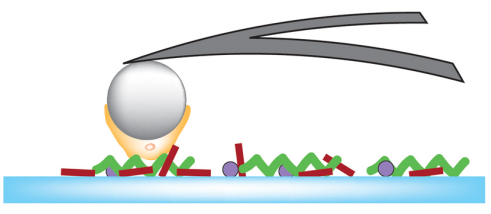

Figure 1. Schematic illustrations of the atomic force microscopy-based biomechanical assays. (A) Indentation experiments were used to characterize the compliance of a single cell. (B) Molecular interactions between a functionalized probe and the membrane receptors were investigated. Single-cell force spectroscopy was utilized to quantify (C) cell-cell adhesions and (D) cell-substrate adhesions. interactions (Fig. 1B), ${ }^{12,13}$ cell-cell interactions (Fig. 1C), ${ }^{14}$ and cell-matrix interactions (Fig. 1D). ${ }^{15}$

Much of a challenge is imposed by the clinical aspect of cancer diagnosis. Although in vitro biomechanical assays have shown that cancer cells were more compliant than normal cells, clinicians typically diagnosed a tumor by sensing an increase in tissue rigidity by palpation, which originates from the matrix-hardening. As a translational research to evaluate the possibility of the biomechanical assays in clinical cancer diagnostics and prognostics, circulating tumor cells (CTCs) harvested from patients' pleural fluids and primary cells derived from cancer patients' biopsy samples have been investigated. ${ }^{16,17}$ In addition, nano-mechanical measurements have been performed for tissues obtained from human and animal model biopsies. ${ }^{18}$ The obtained biomechanical profiles of tissue samples broaden our understanding of clinical aspects of cancer in the tissue integrity. ${ }^{19}$

In this review, we discuss some of recent advances in biomechanical assays in cancer research. We include the studies about in vitro and ex vivo single-cell experiments and in vivo tissue measurements. A possible use of mechanical properties as a measure of anti-cancer drug efficacy tests is also discussed. These nano-mechanical insights will advance our understanding of cancer progression and provide a tool in diagnosing cancer and screening an effective anti-cancer drug with the help of biochemical signatures.

\section{BIOMECHANICS OF CANCER CELLS}

From the material stand point, cells behave like an elastic material. The cellular mechanical compliance, i.e., elastic modulus, defines the ability of cells to deform their shapes and to sustain physiologically relevant stresses. Micropipette aspiration technique is one of the first experiments to characterize mechanical properties of single cells in suspension. ${ }^{20}$ Since the study using micropipette aspiration has reported an increase in deformability of the oncogene-transformed fibroblasts, ${ }^{21}$ various in vitro single cell experimental techniques such as optical tweezers, ${ }^{22}$ optical stretchers, ${ }^{23}$ microplate manipulation, ${ }^{24}$ and magnetic twisting cytometry ${ }^{25}$ have been developed to study mechanical deformability of biological cells. Among these, the AFM-based nano-indentation method is the most widely used technique in determining the mechanical compliance of various cancer cells. ${ }^{26}$

AFM has advantages, such as adaptability to the liquid environment, the ability to measure forces precisely in a localized region, and repeatability. A simple indentation over a localized region of a single cell by an AFM probe yields a force-distance curve with a pico-newton-force and a nano-meter lateral resolution (Fig. 1A). The mathematical models such as the Hertz model have been adapted to calculate the Young's moduli from the obtained force-distance curves. Lower Young's moduli, i.e., elastic moduli, represent higher mechanical deformability of a cell. ${ }^{27}$ With the recent technological advances, force-distance curves can be obtained in two-dimensional arrays so that a local map of mechanical compliances could be delineated over localized cellular regions. ${ }^{7}$ We showed the representative maps of topography (Fig. 2A) and elastic moduli (Fig. 2B) of prostate cancer cells (CL-1), which were reconstructed from the force-distance curves (Fig. 2C) obtained from each pixel of images in two-dimensional array. The nano-indentation technique has been also extended to rheological measurements, which allow us to obtain not only the elastic but also the viscous moduli of cells. ${ }^{28}$ The force-distance curves obtained by the AFM nano-indentation provide quantitative information about the binding events of ligand-receptor interactions in a living cell as well (Fig. 1B). ${ }^{12}$ Further advance in single-cell force spectroscopy utilizing a single-cell attached probe enables quantitative measurements of cell-cell (Fig. 1C) and cell-substrate (Fig. 1D) adhesions under physiological conditions. ${ }^{15}$ The experimental details and previous results using these techniques are beyond 

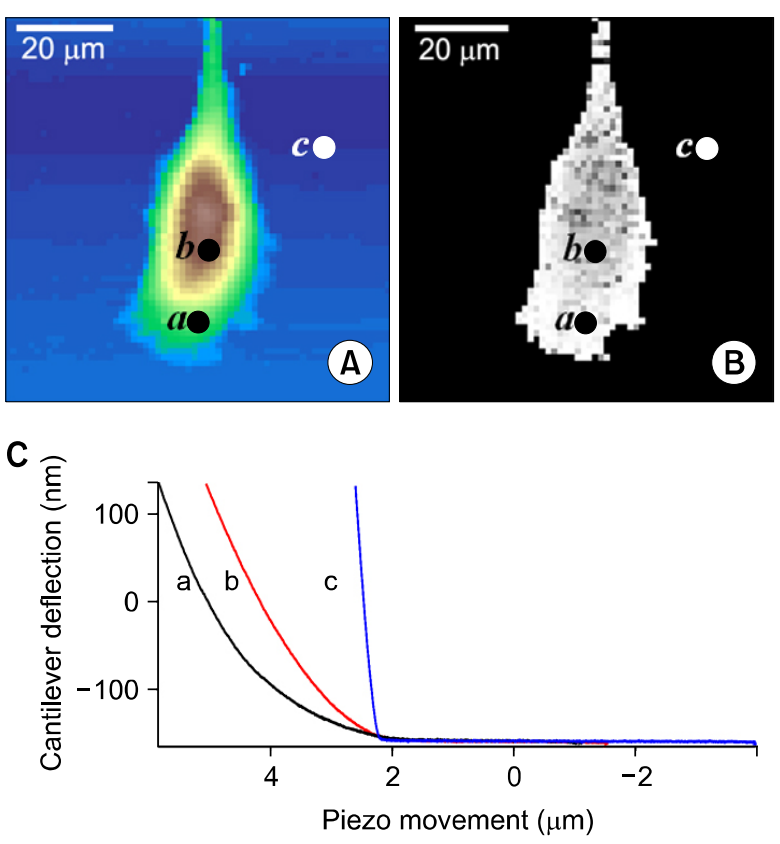

Figure 2. The representative maps of topography and elastic constants determined from force-distance $(f-d)$ curves obtained in two-dimensional arrays. Presented (C) $f-d$ curves were obtained from the points indicated as $a$ (cell edge), $b$ (cell center), $c$ (glass) in the (A) topographic and (B) elastic maps. The horizontal axis represents the $z$-sensor $(\mu \mathrm{m})$ showing the atomic force microscopy (AFM) z-piezo movements while the vertical axis represents the cantilever deflection $(\mathrm{nm})$ of the AFM probe. The $64 \times 64 \mathrm{f}$ - $d$ curves were obtained from the area of $80 \times 80 \mu^{2}$ area.

the scope of this review.

Although the epigenetic and biochemical signatures of cancer cells largely depend on the origin of cancer, in vitro AFM studies have characterized enhanced mechanical compliances as common phenotypic features for cell lines derived from various cancers including bladder, prostate, ovarian, kidney, and breast cancer (Table 1). ${ }^{8.29 .34}$ In order to eliminate the discrepancy originating from the methodological difference in measuring the elastic moduli, we summarize the results obtained only from the AFM nano-indentation. Culture conditions, AFM experimental details such as loading rates and stress regimes, and the analysis details affect the measured values of elastic moduli even from the same cell lines. Thus, it is hard to compare the elastic moduli measured from different study groups. Nevertheless, the data summarized in Table 1 consistently show that the cancerous cells are mechanically more compliant than their normal counterparts in vitro. This suggests that the AFM-based nano-indentation assay can serve as a promising tool to diagnose cancer with no need for specific molecular markers.

\section{ROLE OF CYTOSKELETAL COMPONENTS IN MECHANICAL DEFORMABILITY}

The mechanical compliance of cells is largely governed by the cytoskeleton, three-dimensional network composed of filamentous polymers-actin filaments, microtubules, and intermediate filaments-and associated proteins. The actin filaments were found to be a major contributor for altered mechanical responses from cancer cells. ${ }^{2}$ Although the specific genetic and molecular signatures of cancer vary by the cancer types, alterations in the actin reorganization are commonly observed in all types of cancer. During the cancer formation, actin filaments are reorganized from long and well-organized structures to short and less nucleated or cross-linked structures. Consequently, altered actin organization should result in the enhanced mechanical compliance of cancer cells. In concert with cellular signaling pathways, actin filaments also play an important role in various cellular functions such as mechano-transduction, mitosis, migration, proliferation, adhesion, invasion, and apoptosis. The treatment of cells with actin-disrupting agents such as latraculin A, cytochalasin B, and cytochalain D can induce a remarkable depolymerization of actin filaments and consequently a decrease in the elastic moduli. ${ }^{35}$ The contribution of microtubules to the cellular mechanical stability was also confirmed by treating cells with the tubulin-stabilizing agents such as colcemide and nocodazole, which resulted in a significant increase in mechanical stiffness. ${ }^{36}$

While the roles of actin filaments and microtubules have been well-known, the functions of the intermediate filaments are still under intense investigations. Interestingly, the down-regulation of keratins, intermediate filaments abundant in epithelial cells, during the epithelial-mesenchymal transition is found to be highly correlated with the enhanced migratory and invasive behaviors of tumor cells. ${ }^{37}$ The keratin-free mouse keratinocytes have shown $60 \%$ higher cell deformability and invasiveness. In addition, others have reported that sphingosylphosphorylcholine induces a perinuclear reorganization of keratins and thus a decrease in elastic moduli in pancreatic cancer cells. ${ }^{38}$ On the contrary, vimentins, major intermediate filaments found in mesenchymal cells, play a role in conferring upon cells mechanical compliance and thus invasiveness. ${ }^{39}$ The rheological assay using in vitro filament samples revealed that inter-filament networks composed of both vimentins and actin filaments better contribute to the overall mechanical integrity of cells than the networks containing only actin filaments or only vimentin filaments. ${ }^{40}$ AFM experiments combining confocal fluorescent microscopy 
Table 1. Elastic moduli obtained by AFM-based nano-indentation assays

\begin{tabular}{|c|c|c|c|c|}
\hline Cancer type & Cell line & Cell type & Elastic moduli $(\mathrm{kPa})$ & Reference No. \\
\hline \multirow[t]{5}{*}{ Bladder cancer } & Hu609 & Non-cancerous & $9.7 \pm 3.6$ & 29 \\
\hline & HCV29 & Non-cancerous & $7.5 \pm 3.6$ & \\
\hline & $\mathrm{Hu} 456$ & Cancerous & $0.3 \pm 0.2$ & \\
\hline & $\mathrm{T} 24$ & Cancerous & $0.8 \pm 0.4$ & \\
\hline & BC3726 & Cancerous & $1.0 \pm 3.6$ & \\
\hline \multirow[t]{3}{*}{ Prostate cancer } & $\mathrm{BPH}$ & Non-cancerous & $2.8 \pm 0.5$ & 30 \\
\hline & LNCaP & Lowly metastatic & $0.5 \pm 0.1$ & \\
\hline & PC-3 & Highly metastatic & $1.4 \pm 0.2$ & \\
\hline \multirow[t]{5}{*}{ Ovarian cancer } & IOSE & Non-cancerous & $2.5 \pm 2.0$ & 31 \\
\hline & HEY & Lowly metastatic & $0.9 \pm 0.5$ & \\
\hline & HEY48 & Highly metastatic & $0.5 \pm 0.2$ & \\
\hline & OVCAR-4 & Lowly metastatic & $1.1 \pm 0.9$ & \\
\hline & OVCAR-3 & Highly metastatic & $0.6 \pm 0.2$ & \\
\hline \multirow[t]{3}{*}{ Kidney cancer } & $\mathrm{RC} 124$ & Non-tumorigenic & 9.38 & 32 \\
\hline & A498 & Carcinoma & 7.41 & \\
\hline & ACHN & Adenocarcinoma & 2.48 & \\
\hline \multirow[t]{2}{*}{ Breast cancer } & MCF-10A & Non-cancerous & $1.1 \pm 0.5$ & 33 \\
\hline & MCF-7 & Cancerous & $0.6 \pm 0.2$ & \\
\hline \multirow[t]{2}{*}{ Oral cancer } & UM1 & Highly metastatic & $3.7 \pm 1.0$ & 34 \\
\hline & UM2 & Lowly metastatic & $6.2 \pm 1.4$ & \\
\hline \multirow[t]{3}{*}{ Chondrosarcoma } & JJ012 & Highly metastatic & 0.34 & 8 \\
\hline & FS090 & Moderately metastatic & 1.27 & \\
\hline & $105 \mathrm{KC}$ & Lowly metastatic & 0.79 & \\
\hline
\end{tabular}

Values are presented as mean $\pm \mathrm{SD}$ or mean only. AFM, atomic force microscopy.

and/or a cytoskeleton-affecting agents have been highly utilized to reveal the role of cytoskeletal components in cellular mechanical deformability. ${ }^{41}$ However, the detailed mechanism has yet to be elucidated for better understanding of how each cytoskeletal component interacts with each other and orchestrates cellular signaling pathways affecting cell migration, invasiveness, and proliferation

\section{ROLE OF MECHANICAL DEFORMABILITY IN METASTASIS}

Cancer cells undergo a substantial mechanical deformation during the intra/extra-vasations and tissue invasions. Highly metastatic cancer cells are expected to display enhanced mechanical deformability. Several studies have shown that mechanical deformability is proportionally enhanced as the metastatic potential of cancer cells increases and suggested this enhanced level of deformability as a prognostic as well as a diagnostic marker for cancer. ${ }^{4.5}$ As shown in Table 1, AFM indentation experiments showed a decrease in Young's moduli of highly metastatic ovarian and oral cancer cells. The alteration of the actin organization is reported to be exacerbated with an increase in the metastatic potential and responsible for enhanced mechanical deformability.
However, the reverse or ambiguous correlations between the mechanical compliance and the metastatic potential have been reported from the studies on prostate cancer cells, hepatocellular carcinoma cells, and human chondrosarcoma cell. ${ }^{6.830}$ These results alert a caution against the simple application of the mechanical study to the cancer prognosis. The metastatic cascade begins with a detachment of cancer cells from the epithelium. The subsequent invasion, intra/extra-vasation, and the formation of a secondary tumor involve a dynamic modulation of cell-cell and cell-substrate adhesions as well as a significant level of mechanical deformation. An increasing number of evidence also suggests that the altered mechanical compliance of cancer cells may be caused by a mechano-reciprocal interaction with the microenvironments mediated by the dynamic reorganization of the Focal Adhesions (FAs) ${ }^{42}$ In addition, the intra-cellular calcium dynamics is recognized as an important messenger connecting the long-range coupling between the changes in FAs and mechanical deformability. ${ }^{43}$ Highly metastatic prostate cancer cells show poor mechanical deformability. ${ }^{7}$ This observation requires a more sophisticated explanation because it seems to be contrary to the general prediction about the correlation between mechanical compliance and metastatic potential. AFM force spectroscopic studies combined with fluorescence microscopy have revealed 
that highly metastatic prostate cancer cells show a significant increase in the calcium dynamics, motility, and cell-substrate adhesions. ${ }^{7}$ It is postulated that despite the disadvantage in the tissue invasion due to poor mechanical deformability, above mentioned mechanical signatures can play more important roles in other steps of prostate cancer metastasis. Prostate cancers preferentially metastasize to bones. ${ }^{44}$ It is recognized that tumor cell interactions with the bone microenvironment initiate a vicious cycle that will subsequently lead to a selective growth for the cancer cells and the establishment of osteoclastic or osteoblastic incurable lesions. Enhanced cell-substrate adhesion and calcium dynamics may be favorable for this process. Although the molecular mechanisms underlying tumor-bone interaction have to be identified in detail, multiple mechanical signatures can provide crucial information regarding which steps of metastasis need to be disrupted in order to prevent further metastatic progression.

\section{TRANSLATIONAL APPROACH FOR BIOMECHANICS}

From the results of in vitro biomechanical assays as summarized in Table 1 , it is widely accepted that cancerous cells are more compliant than normal counterparts. It is expected that biomechanical assays can advance the current cancer diagnosis which relies on biological descriptions obtained from morphological observations. Translational approaches have been performed to evaluate the possibility of the clinical use of the biomechanical assays for cancer diagnosis. Recently, ex vivo mechanical analyses using AFM attempted to study mechanical properties of CTCs taken from the pleural fluids of patients with suspected lung, breast, and pancreatic cancer. ${ }^{16}$ From this study, CTCs derived from patients have shown to be more mechanically compliant than benign cells. This behavior suggests that metastasis may be promoted by the mechanical compliance of CTCs. In addition to the AFM experiments, studies using an optical stretcher consistently have shown a soft mechanical signature from oral squamous carcinoma cells derived from oral cancer patients. ${ }^{45}$ Studies using a magnetic tweezer have also reported a power-law correlation between the mechanical compliance with migratory and invasive potential in epithelial ovarian cancer cells isolated from the ascites of patients. ${ }^{17}$ Thus far, ex vivo as well as in vitro studies have provided strong evidence for the enhanced mechanical compliance in cancer cells.

Nevertheless, the clinical application of biomechanical assays as a cancer diagnostic tool is considered to be still primitive because of long-standing ambiguities attributed from the mechano-reciprocity between cancer cells and tumor microenvironments. Common clinical wisdom by palpation recognizes a relative stiffening of tumor than the surrounding tissue. This notion seems to be paradoxical to the results obtained from single cell mechanical assays, which revealed the soft mechanical nature of cancer cells compared to the healthy counterparts. According to the mechano-reciprocity, the two-way communication between cancer cells and the extracellular matrix (ECM) stimulates the Rho/ROCK pathway and promotes an increase in the ECM stiffness and cell contractility mediated by focal adhesions, i.e., integrin clusters. ${ }^{46}$ Thus, it is of paramount interest to elucidate the biomechanical heterogeneity of tumor tissues originated from soft cancer cells and stiff ECM. AFM-based nano-indentation experiments on human breast biopsied tissues have revealed that the nano-mechanical compliance of malignant tissues shows a broader distribution resulting from tissue heterogeneity compared to normal and benign tissues. ${ }^{18}$ A prominent low-stiffness peak in the distribution is postulated to represent the soft nature of cancer cells. They have also provided similar findings obtained from MMTV-PyMT transgenic mice and lungs of mice with late-stage tumor. Tian et al., ${ }^{47}$ also evaluated whether the nano-mechanical profiles could help define the stage of hepatocellular carcinoma progression. They reported a further downward shift of the lowest elasticity peak as the cirrhotic tissues progressed to a malignant state. This enhanced mechanical compliance was considered to be highly correlated with microvascular invasion. Interestingly, a Rho-family effector (mDial) was noticed as a molecular origin of this mechanical change found in liver tissues. Similar mechanical softening has been also reported from tissue samples obtained from endometrioid carcinoma of the uterine corpus, breast cancer, and vulvar carcinoma. ${ }^{48}$ These results together imply that the nanomechanical profiles of tissue samples obtained from various types of cancer reflect the heterogeneous nature of the mechanical compliance due to the complex composition of tissues and the lowest peak of elasticity may coincide with mechanical properties identified from the in vitro single-cell biomechanical assay.

\section{MECHANICAL ASPECTS OF ANTI-CANCER DRUG RESISTANCE}

Several causes responsible for anti-cancer drug resistance have been identified. Alterations in apoptotic pathway, induction of drug-detoxifying mechanism, and aberrant regulation of drug transporters that reduces the accumulation of drugs by ejection 
from the cells have been suggested previously. ${ }^{49.50}$ Certain anti-cancer drugs are designed to modulate cell membranes and cytoskeletons in order to induce cytotoxic action. ${ }^{2}$ For example, paclitaxel abnormally reinforces the microtubule organization by inducing polymerization and suppressing depolymerization. Vincristin and vinblastine also modulate the microtubule organization and suppress mitosis. Lam et al., ${ }^{51}$ also have shown from the AFM experiments that the chemotherapeutic treatment using dexamethasone or daunorubicin induced mechanical stiffening of leukemia cells and caused the vascular complications in acute leukemia. When the actin cytoskeleton is disrupted by cytochalasin $\mathrm{D}$, such mechanical stiffening after the chemotherapeutic treatment is significantly suppressed. However, the significance of mechanical alterations on the acquisition of anti-cancer drug resistance is not corroborated until recently.

Recent AFM experiments performed on ovarian cancer cells have attempted to reveal whether cisplatin resistance correlates with the dynamic reorganization of actin filaments and the nano-mechanical compliance. ${ }^{10.52}$ While the cisplatin-sensitive cells show dose-dependent cell stiffening after the cisplatin treatment, cisplatin treatment does not induce a nano-mechanical modulation in resistant cells. In addition, by the stimulated emission depletion microscopy, researchers have found distinct differences in actin structures between the drug-sensitive and resistant cells. While the drug-sensitive cells showed scattered and non-oriented filaments, the drug-resistant cells have highly organized actin filaments. From the experiments using nine different ovarian cancer cell lines, a linear correlation between cisplatin resistance measured by cell viability assay and Young's moduli was confirmed. ${ }^{11}$ The actin-disrupting agents are also used to identify the actin dynamics as a major contributor responsible for such correlation. A well-organized actin cytoskeleton may physically block the anti-cancer drug uptake. However, the molecular mechanism on how cell stiffening accompanied by the strengthened actin organization affects the drug sensitivity has to be better elucidated. Interestingly, people have shown that Rho GTPase directly controls the dynamic reorganization of actin cytoskeletons and cell stiffness and thus affects the cisplatin-sensitivity. The Rho mediated pathway regulates many cellular functions such as cell polarity, gene transcription, and microtubule dynamics. More detailed investigations have yet to be performed to understand the correlation between the anti-cancer drug resistance and cytoskeletal alterations. Nevertheless, nano-mechanical stiffness of cancer cells can provide a useful insight for pre-evaluating the anti-cancer drug efficacy and designing a personalized chemotherapeutic strategy.

\section{CONCLUSION}

Since nano-mechanical assay was introduced to cancer biology, the mechanical alteration found in cancer cells in vitro was commonly observed in various cancers. Change in actin cytoskeleton was noticed as a major cause responsible for such mechanical alterations in cancer cells. The role of other filamentous components such as keratin and vimentin and the interconnected functions between actin and other filaments are still under investigations. The translational studies to interrogate the possibility to use the AFM-based indentation experiments in the clinical setting have been tried using the CTCs and human biopsied tissues. Although the mechanical stiffening of extracellular matrix due to the mechano-reciprocal interaction seemed to provide the complications for nano-mechanical profiling of biopsied tissues, it can be concluded that the enhanced softness might be a common feature of cancer cells not only from in vitro but also from in vivo studies. In addition, the nano-mechanical reinforcements observed in cancer cells by the anti-cancer drug treatment should provide quantitative information to determine which chemotherapeutic agents will cure the cancer more efficiently under personalized conditions.

\section{ACKNOWLEDGMENTS}

This research was supported by Basic Science Research Program through the National Research Foundation of Korea (NRF) funded by the Ministry of Science, ICT and Future Planning (2013R1A1A3008608).

\section{CONFLICTS OF INTEREST}

No potential conflicts of interest were disclosed.

\section{REFERENCES}

1. Wirtz D, Konstantopoulos K, Searson PC. The physics of cancer: the role of physical interactions and mechanical forces in metastasis. Nat Rev Cancer 2011;11:512-22.

2. Suresh S. Biomechanics and biophysics of cancer cells. Acta Mater 2007;55:3989-4014.

3. Lekka M, Pogoda K, Gostek J, Klymenko O, Prauzner-Bechcicki S, Wiltowska-Zuber J, et al. Cancer cell recognition--mechanical phenotype. Micron 2012;43:1259-66.

4. Guck J, Schinkinger S, Lincoln B, Wottawah F, Ebert S, Romeyke $\mathrm{M}$, et al. Optical deformability as an inherent cell marker for testing 
malignant transformation and metastatic competence. Biophys J 2005;88:3689-98.

5. Ochalek T, Nordt FJ, Tullberg K, Burger MM. Correlation between cell deformability and metastatic potential in B16-F1 melanoma cell variants. Cancer Res 1988;48:5124-8.

6. Zhang G, Long M, Wu ZZ, Yu WQ. Mechanical properties of hepatocellular carcinoma cells. World J Gastroenterol 2002;8:243-6.

7. Bastatas L, Martinez-Marin D, Matthews J, Hashem J, Lee YJ, Sennoune S, et al. AFM nano-mechanics and calcium dynamics of prostate cancer cells with distinct metastatic potential. Biochim Biophys Acta 2012;1820:1111-20.

8. Darling EM, Zauscher S, Block JA, Guilak F. A thin-layer model for viscoelastic, stress-relaxation testing of cells using atomic force microscopy: do cell properties reflect metastatic potential? Biophys J 2007:92:1784-91.

9. Moore NM, Nagahara LA. Physical biology in cancer. 1. Cellular physics of cancer metastasis. Am J Physiol Cell Physiol 2014;306:C78-9.

10. Seo YH, Jo YN, Oh YJ, Park S. Nano-mechanical reinforcement in drug-resistant ovarian cancer cells. Biol Pharm Bull 2015;38:389-95.

11. Sharma S, Santiskulvong C, Rao J, Gimzewski JK, Dorigo O. The role of Rho GTPase in cell stiffness and cisplatin resistance in ovarian cancer cells. Integr Biol (Camb) 2014;6:611-7.

12. Hinterdorfer P, Dufrêne YF. Detection and localization of single molecular recognition events using atomic force microscopy. Nat Methods 2006;3:347-55.

13. Fisher TE, Carrion-Vazquez M, Oberhauser AF, Li HB, Marszalek PE, Fernandez JM. Single molecular force spectroscopy of modular proteins in the nervous system. Neuron 2000;27:435-46.

14. Helenius J, Heisenberg CP, Gaub HE, Muller DJ. Single-cell force spectroscopy. J Cell Sci 2008;121:1785-91.

15. Friedrichs J, Helenius J, Muller DJ. Quantifying cellular adhesion to extracellular matrix components by single-cell force spectroscopy. Nat Protoc 2010;5:1353-61.

16. Cross SE, Jin YS, Rao J, Gimzewski JK. Nanomechanical analysis of cells from cancer patients. Nat Nanotechnol 2007;2:780-3.

17. Swaminathan V, Mythreye K, O'Brien ET, Berchuck A, Blobe GC, Superfine R. Mechanical stiffness grades metastatic potential in patient tumor cells and in cancer cell lines. Cancer Res 2011;71:5075-80.

18. Plodinec M, Loparic M, Monnier CA, Obermann EC, Zanetti-Dallenbach R, Oertle $\mathrm{P}$, et al. The nanomechanical signature of breast cancer. Nat Nanotechnol 2012;7:757-65.

19. Hanahan D, Weinberg RA. Hallmarks of cancer: the next generation. Cell 2011;144:646-74.

20. Evans EA. Bending elastic modulus of red blood cell membrane derived from buckling instability in micropipet aspiration tests. Biophys J 1983:43:27-30.

21. Ward KA, Li WI, Zimmer S, Davis T. Viscoelastic properties of transformed-cells - role in tumor-cell progression and metastasis formation. Biorheology 1991;28:301-13.

22. Hénon S, Lenormand G, Richert A, Gallet F. A new determination of the shear modulus of the human erythrocyte membrane using optical tweezers. Biophys J 1999;76:1145-51.

23. Guck J, Ananthakrishnan R, Mahmood H, Moon TJ, Cunningham CC, Käs J. The optical stretcher: a novel laser tool to micromanipulate cells. Biophys J 2001;81:767-84.

24. Thoumine O, Ott A. Time scale dependent viscoelastic and contractile regimes in fibroblasts probed by microplate mani- pulation. J Cell Sci 1997;110:2109-16.

25. Wang N, Ingber DE. Probing transmembrane mechanical coupling and cytomechanics using magnetic twisting cytometry. Biochem Cell Biol 1995:73:327-35.

26. Lekka M, Laidler P. Applicability of AFM in cancer detection. Nature Nanotechnol 2009:4:72; author reply 72-3.

27. Park S, Lee YJ. AFM-based dual nano-mechanical phenotypes for cancer metastasis. J Biol Physics 2014:40:413-9.

28. Park S, Koch D, Cardenas R, Käs J, Shih CK. Cell motility and local viscoelasticity of fibroblasts. Biophys J 2005;89:4330-42.

29. Lekka M, Laidler P, Gil D, Lekki J, Stachura Z, Hrynkiewicz AZ. Elasticity of normal and cancerous human bladder cells studied by scanning force microscopy. Eur Biophys J 1999;28:312-6.

30. Faria EC, Ma N, Gazi E, Gardner P, Brown M, Clarke NW, et al. Measurement of elastic properties of prostate cancer cells using AFM. Analyst 2008; 133:1498-500.

31. Xu W, Mezencev R, Kim B, Wang L, McDonald J, Sulchek T. Cell stiffness is a biomarker of the metastatic potential of ovarian cancer cells. PLoS One 2012; 7:e46609.

32. Rebelo LM, de Sousa JS, Mendes Filho J, Radmacher M. Comparison of the viscoelastic properties of cells from different kidney cancer phenotypes measured with atomic force microscopy. Nanotechnology 2013;24:055102.

33. Li QS, Lee GY, Ong CN, Lim CT. AFM indentation study of breast cancer cells. Biochem Biophys Res Commun 2008;374:609-13.

34. Zhou Z, Zheng C, Li S, Zhou X, Liu Z, He Q, et al. AFM nanoindentation detection of the elastic modulus of tongue squamous carcinoma cells with different metastatic potentials. Nanomedicine 2013;9:864-74.

35. Rotsch C, Radmacher M. Drug-induced changes of cytoskeletal structure and mechanics in fibroblasts: an atomic force microscopy study. Biophys 2000;78:520-35.

36. Wu HW, Kuhn T, Moy VT. Mechanical properties of L929 cells measured by atomic force microscopy: effects of anticytoskeletal drugs and membrane crosslinking. Scanning 1998;20:389-97.

37. Seltmann K, Fritsch AW, Käs JA, Magin TM. Keratins significantly contribute to cell stiffness and impact invasive behavior. Proc Nat Acad Sci U S A 2013;110:18507-12.

38. Park MK, Park S, Kim HJ, Kim EJ, Kim SY, Kang GJ, et al. Novel effects of FTY720 on perinuclear reorganization of keratin network induced by sphingosylphosphorylcholine: involvement of protein phosphatase 2A and G-protein-coupled receptor-12. Eur J Pharmacol 2016;775:86-95.

39. Rathje LSZ, Nordgren N, Pettersson T, Rönnlund D, Widengren J, Aspenström $\mathrm{P}$, et al. Oncogenes induce a vimentin filament collapse mediated by HDAC6 that is linked to cell stiffness. Proc Nat Acad Sci U S A 2014;111:1515-20.

40. Esue O, Carson AA, Tseng Y, Wirtz D. A direct interaction between actin and vimentin filaments mediated by the tail domain of vimentin. J Biol Chem 2006;281:30393-9.

41. Haga H, Sasaki S, Kawabata K, Ito E, Ushiki T, Sambongi T. Elasticity mapping of living fibroblasts by AFM and immunofluorescence observation of the cytoskeleton. Ultramicroscopy 2000;82:253-8.

42. Discher DE, Janmey P, Wang YL. Tissue cells feel and respond to the stiffness of their substrate. Science 2005;310:1139-43.

43. Wei C, Wang X, Chen M, Ouyang K, Song LS, Cheng H. Calcium flickers steer cell migration. Nature 2009;457:901-5.

44. Bubendorf L, Schöpfer A, Wagner U, Sauter G, Moch H, Willi N, et 
al. Metastatic patterns of prostate cancer: an autopsy study of 1,589 patients. Hum Pathol 2000;31:578-83.

45. Remmerbach TW, Wottawah F, Dietrich J, Lincoln B, Wittekind C, Guck J. Oral cancer diagnosis by mechanical phenotyping. Cancer Res 2009;69:1728-32.

46. Huang S, Ingber DE. Cell tension, matrix mechanics, and cancer development. Cancer Cell 2005;8:175-6.

47. Tian M, Li Y, Liu W, Jin L, Jiang X, Wang X, et al. The nanomechanical signature of liver cancer tissues and its molecular origin. Nanoscale 2015;7:12998-3010.

48. Lekka M, Gil D, Pogoda K, Dulińska-Litewka J, Jach R, Gostek J, et al. Cancer cell detection in tissue sections using AFM. Arch Biochem Biophys 2012;518:151-6.

49. Shen DW, Pouliot LM, Hall MD, Gottesman MM. Cisplatin resist- ance: a cellular self-defense mechanism resulting from multiple epigenetic and genetic changes. Pharmacol Rev 2012;64:706-21.

50. Parker RJ, Eastman A, Bostick-Bruton F, Reed E. Acquired cisplatin resistance in human ovarian cancer cells is associated with enhanced repair of cisplatin-DNA lesions and reduced drug accumulation. J Clin Invest 1991:87:772-7.

51. Lam WA, Rosenbluth MJ, Fletcher DA. Chemotherapy exposure increases leukemia cell stiffness. Blood 2007;109:3505-8.

52. Sharma S, Santiskulvong C, Bentolila LA, Rao J, Dorigo O, Gimzewski JK. Correlative nanomechanical profiling with super-resolution F-actin imaging reveals novel insights into mechanisms of cisplatin resistance in ovarian cancer cells. Nanomedicine 2012;8:757-66. 